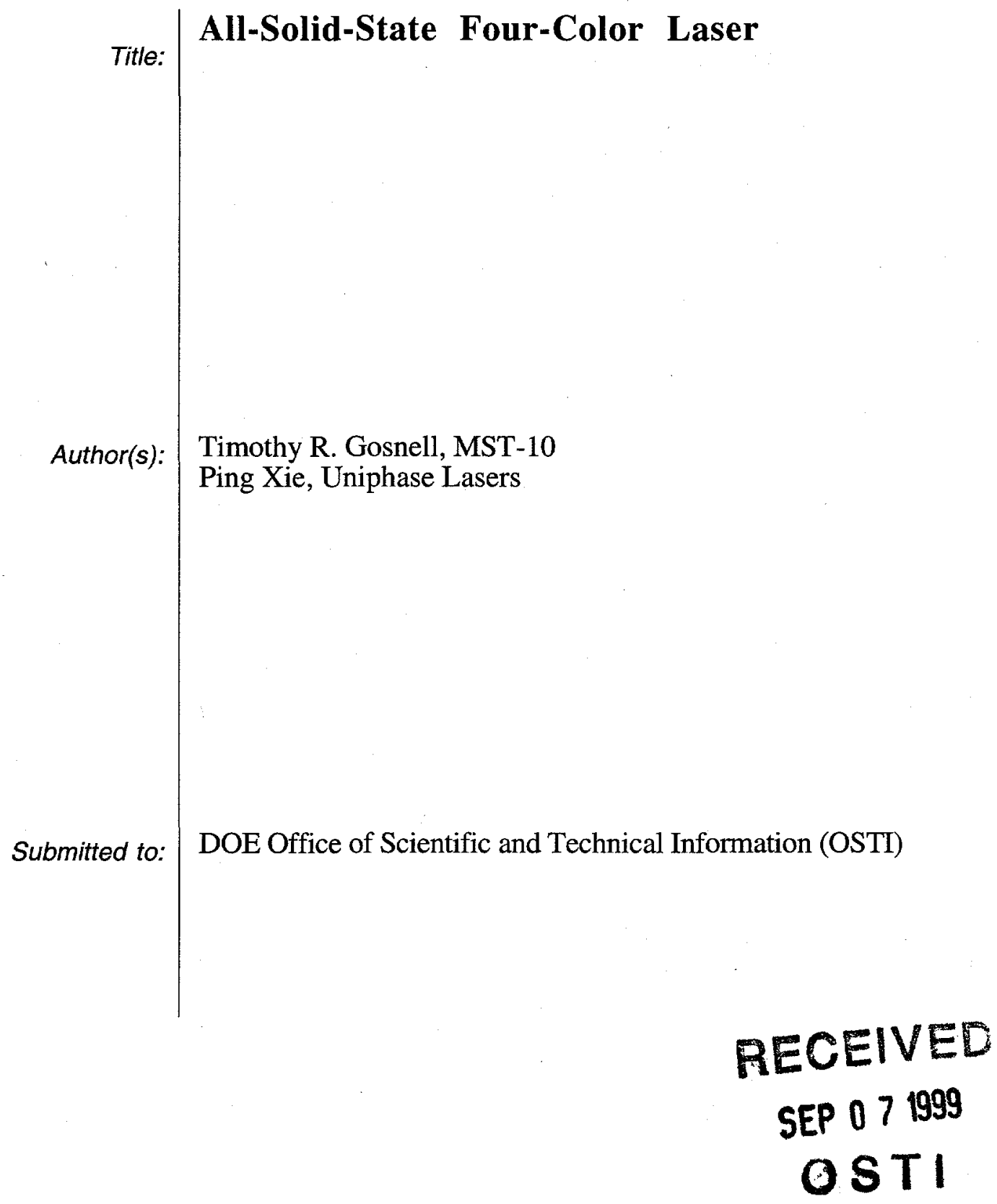

Los Alamos National Laboratory, an affirmative action/equal opportunity employer, is operated by the University of California for the U.S. Department of Energy under contract W-7405-ENG-36. By acceptance of this article, the publisher recognizes that the U.S. Government retains a nonexclusive, royaltyfree license to publish or reproduce the published form of this contribution, or to allow others to do so, for U.S. Government purposes. Los Alamos National Laboratory requests that the publisher identify this article as work performed under the auspices of the U.S. Department of Energy. Los Alamos National Laboratory strongly supports academic freedom and a researcher's right to publish; as an institution, however, the Laboratory does not endorse the viewpoint of a publication or guarantee its technical correctness. 


\section{DISCLAIMER}

This report was prepared as an account of work sponsored by an agency of the United States Government. Neither the United States Government nor any agency thereof, nor any of their employees, make any warranty, express or implied, or assumes any legal liability or responsibility for the accuracy, completeness, or usefulness of any information, apparatus, product, or process disclosed, or represents that its use would not infringe privately owned rights. Reference herein to any specific commercial product, process, or service by trade name, trademark, manufacturer, or otherwise does not necessarily constitute or imply its endorsement, recommendation, or favoring by the United States Government or any agency thereof. The views and opinions of authors expressed herein do not necessarily state or reflect those of the United States Government or any agency thereof. 


\section{DISCLAIMER}

Portions of this document may be illegible in electronic image products. Images are produced from the best available original document. 


\title{
All-Solid-State Four-Color Laser
}

\author{
*Timothy R. Gosnell (MST-10) \\ Ping Xie (Uniphase Lasers)
}

\begin{abstract}
This is the final report of a three-year, Laboratory Directed Research and Development (LDRD) project at Los Alamos National Laboratory (LANL). The goal of this project is to develop a solid state laser that produces visible output wavelengths, including the commercially compelling blue wavelength. The basic architecture of the device consists of a single-mode optical fiber doped with $\mathrm{Pr}^{3+}$ and $\mathrm{Yb}^{3+}$ ions. When the ions are simultaneously pumped with a near infrared laser $(860 \mathrm{~nm})$, complex energy transfer processes involving multiple excited ions leads to population of a high-lying energy level of $\mathrm{Pr}^{3+}$. Results include the demonstration of the existence of a photon avalanche mechanism responsible for creation of the population inversion and demonstration of the highest optical-to-optical efficiency of any up-conversion laser reported to date. A US Patent was awarded for this invention in 1998.
\end{abstract}

\section{Background and Research Objectives}

Everyone knows what a megabtye is and complains about not having enough of them. Consumer demand for digital mass-storage devices thus currently makes for a $\$ 50$ billion per year industry; by all accounts, this demand will soar when the information-based economies of the next century begin to emerge. Optical memory materials offer the greatest technological leverage we know of for rapidly increasing the number of megabytes storable on one square inch of mass-storage recording medium. In turn, the best strategy for increasing the density of information recordable on optical materials is to shorten the operating wavelength of the storage device's laser read-write heads. Areal optical storage density scales as the inverse square of the operating wavelength, hence blue- or uv-based optical disk drives promise a four- to six-fold expansion in memory capacity over the infrared optical drives available today. Fifty gigabytes of data could fit onto a single twoinch disk written at $450 \mathrm{~nm}$; even terabyte storage capacities will become practical pending the development of multilayer/multiwavelength optical recording materials.

*Principal Investigator, e-mail: gosnell@ lanl.gov 
In July, 1994, we discovered a solid-state energy-transfer mechanism-known generically as a photon avalanche-that has allowed us to demonstrate the first all-solidstate laser capable of generating multicolor visible output. It is the first all-solid-state laser to directly generate blue wavelengths ${ }^{1}$ at room temperature; at the time of its discovery it produced the highest output power at the highest efficiency of any "up-conversion" laser demonstrated to date; and it is the first commercially practical laser of any type to produce all three primary colors. The laser produces red $(635 \mathrm{~nm})$, orange $(615 \mathrm{~nm})$, green $(520$ $\mathrm{nm})$, and blue $(491 \mathrm{~nm})$ wavelengths, is modestly tunable, and operates at room temperature. It is optically pumped with a single off-the-shelf infrared diode laser. Aside from applications in uv, blue, and multi-wavelength "super-resolution" optical disk drives, other compelling applications for the device include broad-spectrum chemical sensors, process monitoring, flow cytometry, photodynamic therapy, gene sequencing, highresolution color reprographics, and high-brightness full-color projectors.

The Laboratory's Intellectual Property Group submitted a patent application for our invention to the US Patent Office in September, 1994. This LDRD project further investigates the population dynamics of the laser's gain medium and undertakes a multitude of optical engineering experiments intended to optimize the performance of the device. The salient objectives of the project are to demonstrate $1000 \mathrm{~mW}$ of output in the red, at least $100 \mathrm{~mW}$ of output in the green, at least $50 \mathrm{~mW}$ of output in the blue, and, by adding a harmonic-conversion crystal to the red laser, to demonstrate at least $10 \mathrm{~mW}$ of output in the ultraviolet.

The laser consists of a 25-centimeter section of a single-mode, fluoride-glass optical-fiber waveguide whose core has been doped with triply charged praseodymium and ytterbium ions. A commercial 860-nm AlGaAs semiconductor-diode laser is used as the optical pump source. The balance of the design consists of simple passive components. Wavelength-selective dielectric mirrors that favor reflection of one of the four output colors are butted against the fiber's ends in order to form the laser's optical cavity, while a small aspheric lens is used to focus the near-infrared diode-laser pump beam through one of these end mirrors onto the fiber's light-guiding core.

The fiber emits visible light because energy resident in two infrared pump photons can accumulate onto a single $\mathrm{Pr}^{3+}$ ion. In brief, photon-avalanche optical pumping begins

\footnotetext{
${ }^{1}$ II-VI semiconductor injection lasers producing blue wavelengths have been previously reported, but their continuous operating lifetimes at room temperature have never exceeded one minute. Even if their damage mechanisms are understood and overcome, it will be years béfore a commercially feasible device will be demonstrated.
} 
when a single, highly excited $\mathrm{Pr}^{3+}$ ion in the ${ }^{3} \mathrm{P}_{0}$ upper laser level sacrifices some of its energy to a neighboring unexcited $\mathrm{Yb}^{3+}$ ion. Radiationless energy transfer from the newly excited $\mathrm{Yb}^{3+}$ ion to a second $\mathrm{Pr}^{3+}$ yields a total of two $\mathrm{Pr}^{3+}$ ions in the ${ }^{1} \mathrm{G}_{4}$ level, a state located approximately midway between the $\mathrm{Pr}^{3+}$ ground state and the ${ }^{3} \mathrm{P}_{0}$ upper laser level. By tuning the pump laser to the ${ }^{1} \mathrm{G}_{4} \rightarrow{ }^{3} \mathrm{P}_{0}$ transition, however, excited-state absorption promotes both $\mathrm{Pr}^{3+}$ ions to the upper laser level where only one ion resided before. The process repeats and in perhaps $100 \mu$ s generates an excited $\mathrm{Pr}^{3+}$ population large enough to yield optical gain above threshold.

All that is required to trigger this photon-avalanche pumping mechanism is to generate by some means a tiny seed population in the $\mathrm{Pr}^{3+}{ }^{3} \mathrm{P}_{0}$ state. The co-doped $\mathrm{Yb}^{3+}$ ions perform this function. By weakly absorbing pump light at the extreme shortwavelength limits of the $\mathrm{Yb}^{3+} 980-\mathrm{nm}$ ground-state absorption band, the few $\mathrm{Yb}^{3+}$ ions excited in this way serve as energy donors to the $\operatorname{Pr}^{3+}{ }^{1} G_{4}$ level. Excited-state absorption on the ${ }^{1} \mathrm{G}_{4} \rightarrow{ }^{3} \mathrm{P}_{0}$ transition is enough to produce the required seed population. As should be surmised, an energy-level diagram depicting these processes would be nearly incomprehensible.

Multiple laser output wavelengths are observed from the fiber because of praseodymium's rich energy-level structure below the upper laser level. Red output is

produced by the ${ }^{3} \mathrm{P}_{0} \rightarrow{ }^{3} \mathrm{~F}_{2}$ transition, orange by the ${ }^{3} \mathrm{P}_{0} \rightarrow{ }^{3} \mathrm{H}_{6}$ transition, long green output at $535 \mathrm{~nm}$ is produced by the ${ }^{3} \mathrm{P}_{0} \rightarrow{ }^{3} \mathrm{H}_{5}$ transition, short green output is produced at 520 nm by the ${ }^{3} \mathrm{P}_{1} \rightarrow{ }^{3} \mathrm{H}_{5}$ transition (note that ${ }^{3} \mathrm{P}_{1}$ is thermally populated from the ${ }^{3} \mathrm{P}_{0}$ level), and finally blue output is produced on the ${ }^{3} \mathrm{P}_{0} \rightarrow{ }^{3} \mathrm{H}_{4}$ transition.

\section{Importance to LANL's Science and Technology Base and National R\&D Needs}

Gas lasers that produce visible output wavelengths are bulky, unreliable, expensive, and extraordinarily inefficient. The impasse such disadvantages present to the development of commodity products like digital optical disk drives now motivates an intense international competition to demonstrate and market all-solid-state visible sources. Our discovery of the photon-avalanche mechanism and its effectiveness in producing useful visible lasers have thrust us into this competition. Within the research community, at stake is federal sponsorship of visible source development. An award of \$1.45 million from the 
Department of Commerce Advanced Technology Program was just made to the Uniphase Corporation for development of blue frequency-doubled "micro-chip" lasers. The Advanced Research Projects Agency is funding the Optical Materials Center, a multiinstitutional program devoted in part to basic research on solid-state blue and green laser sources. Within the business community, at stake are enormous commercial profits. Industry participants at a recent OMC workshop included Xerox, Hewlett Packard, New Focus, Liconix, Rockwell International, Kodak, Hughes Aircraft, Coherent, Uniphase, Union Carbide, Spectra Diode Labs, IBM, Corning, and Texas Instruments. Here's why:

\section{Applications in Chemical Sensing/Process Monitoring}

Low product yield is the bete noire of the semiconductor industry, hence methods for detecting flawed chips well before packaging and testing promise huge increases in productivity. A major application of blue and uv sources is in-line inspection for microscopic defects on semiconductor microelectronics wafers. For chemical sensing, the holy grail of general-purpose Raman instruments is a $750 \mathrm{~nm}$ source, yet niche applications exist for multicolor sources, especially those with high output powers. For example, Kaiser Optical provides green-based Raman spectrometers to the polymer and pharmaceutical industries. In sensing applications relevant to the DOE, short wavelength sources make possible detection of uranyl compounds by laser-induced fluorescence; for efforts in environmental remediation, a specific application is the discrimination of uranyl phosphates from hydroxides.

\section{Applications in Biology and Biomedicine}

Development of a high-power, solid-state orange source will make possible the commercialization of instruments for photodynamic therapy, a technique by which cancer cells are thermally destroyed by a special cell-selective dye that absorbs only at $615 \mathrm{~nm}$. Measurement of lymphocyte ratios for the treatment AIDS is based on 488-nm laser flow cytometry; flow cytometers for disease diagnosis and cell counting are employed in 3000 regional medical centers world wide. Substitution of new solid-state sources for the aircooled argon lasers found universally in these machines will make them more reliable, less expensive, easier to maintain, expand their potential market, and therefore allow them to reach more patients. At Los Alamos, the Life Sciences Division is constructing for the US Army an ultracompact flow cytometer for detection of biowarfare agents in the field. A 50$\mathrm{mW}$ source at $490 \mathrm{~nm}$ is used in this application. For the DOE's Human Genome programs ongoing at Los Alamos, low-resolution sizing of RNA and DNA fragments requires a continuous-wave $50-\mathrm{mW}$ green source, while high-resolution fragment sizing and especially gene sequencing require a mode-locked (short-pulse) green source in the 10$50 \mathrm{~mW}$ power range. A mode-locked version of the $\mathrm{Pr}^{3+}$ fiber laser's green output is 
perfect for this application; pulses as short as $\mathbf{5 0} \mathrm{fs}$ are supportable by the laser's green tuning range.

\section{Applications in High-Resolution Reprographics}

The Kodak Company manufactures color laser printers and copiers that currently employ three separate laser heads. The $\mathrm{Pr}^{3+}$ laser could serve as a single-element replacement in this application by multiplexing of the laser's output wavelengths- the required output powers are already in hand. Short-wavelength lasers are also used for writing of high-resolution printer plates.

\section{Applications in Femtosecond Scientific Lasers}

The laser spectroscopy community despises argon-ion lasers. Nevertheless, argonpumped Ti:sapphire lasers are the tools of choice for obtaining stable, high-power, and tunable ultrashort optical pulses. The extraordinary efficiency and high power of the $\mathrm{Pr}^{3+}$ fiber laser operating in the red, plus the laser's near-diffraction-limited beam quality, makes feasible an all-solid-state femtosecond laser that could displace Ti:sapphire in all but a few applications. A new laser gain medium- $\mathrm{Cr}^{3+}$-doped $\mathrm{LiSrGaF}_{6}-$ possesses a tuning band that overlaps that of Ti:sapphire and a red pump-absorption band perfectly matched to the $\mathrm{Pr}^{3+}$ laser's red output wavelength. In the long run, it is possible that output powers in the green high enough to pump a mode-locked Ti:sapphire laser will be achieved. Such devices would be significantly less expensive to purchase and maintain than an argon/Ti:sapphire system; the single drawback would be somewhat reduced bandwidth. Allsolid-state femtosecond lasers would find applications within the DOE complex that include optical diagnostics for the National Magnetic Field Facility, development of "fast-ignitor" concepts for inertial confinement fusion, and AGEX studies of shock-waves and highenergy-density physics.

\section{Applicatons in Data Storage}

This is the BIG application for short-wavelength lasers. Since the inception of this project, the Accelerated Scientific Computing Initiative has evolved at LANL into a major scientific program. The program's emphasis on ultra-high-speed numeric computation that will ultimately involve terabyte and even petabyte data sets means that mass storage devices of huge capacity will be essential to the success of the program. The blue output of the $\mathrm{Pr}^{3+}$ laser is already adequate for archival read-only systems; a full read-write system requires increasing the blue output power to $30 \mathrm{~mW}$. Frequency doubling of the red output into the ultraviolet should make feasible even higher-resolution read-write drives. Next to power and amplitude noise, the most important specification for a read-write optical-storage laser is its beam quality. Fiber lasers are unsurpassed in this regard because single- 
transverse-mode optical waveguides have no choice but to emit diffraction-limited output beams. But the cost of diode pump lasers dominates the discussion of how deeply shortwavelength optical data storage will penetrate the marketplace; high sales volumes must be assured before the cost of diode lasers fall to meet the $\$ 1000$ ceiling on source costs for PC optical disk drives.

\section{Applications in Full-Color Projection Displays}

The $\mathrm{Pr}^{3+}$ laser has already demonstrated adequate output power for head-mounted virtual-reality displays and virtual cockpits for military aircraft. A projection television or full-color holographic display would require perhaps $100 \mathrm{~mW}$ per primary color, while a 30 -ft flight-simulator dome would require 1 watt per color. Texas Instruments is developing an astonishing ancillary component needed for these applications: a monolithically integrated multi-beam angular scanning chip that when combined with an amplitude modulator for the laser power could project a full-color moving image.

\section{Scientific Approach and Accomplishments}

The year-by-year scientific approach as conceived at the beginning of the project appears immediately below followed by a discussion of the technical results.

First year: Dynamics studies, fiber design, output coupling experiments. The complete details of the photon-avalanche process have yet to be fully elucidated. In particular, there is evidence for a saturated energy-transfer step somewhere in the population dynamics chain but its location and influence on the pumping efficiency is unknown. Discovering the identity of this step is likely to suggest ideas for increasing the pumping rate. To this end, a custom laser confocal microscope will be designed that will allow investigation of population dynamics in the regime of high pump intensity. In order to scale up the output power, a multi-cladded fiber must be designed and fabricated in order to accommodate the reduced beam quality of multi-watt diode laser bars. At fixed pump power, the laser's output power at all four wavelengths must be optimized with respect to output coupler reflectivity. The most straightforward, but expensive, technique for accomplishing this task is to evaporate dielectric mirrors directly onto the fiber end faces and iterate until the output power is maximized. These experiments may need to be repeated for gross increases in pump power. Frequency doubling of the red output will be attempted.

Second year: Mathematical model, high-power pumping, temperature dependence, amplitude and frequency stabilization. Basic investigations of the photon-avalanche population dynamics carried out in the first year will be incorporated into a computational model of the laser's behavior; the objective is to develop design criteria for 
scaling of the laser's output power. Guided by this model, high-power pumping experiments will be performed on a multi-cladded fiber by using as a pump laser either a high-power diode bar or a cw Ti:sapphire laser used as a diode-laser surrogate. Because the electronic transition for blue output terminates on the $\mathrm{Pr}^{3+}$ ground-state Stark manifold, the gain at blue wavelengths is extremely sensitive to the temperature of the active core. Cooling of the fiber with no more than a conventional thermoelectric cooler could improve the performance of the blue laser several fold, especially at high pump powers. An important specification for many of the sensing applications is amplitude stability. Both passive and active means for stabilizing the output power will be investigated. For spectroscopic applications, techniques for wavelength tuning, bandwidth narrowing, and frequency stabilization are needed. Possible choices include scanning prisms, birefringent filters, and intracavity Fabry-Perot filters. Stress-induced birefringence in the fiber itself is a previously unexplored mechanism for tuning of a fiber laser's output.

\section{Third year: Wavelength multiplexing, direct mode locking,}

femtosecond LiSGAF experiments. For wavelength multiplexing the question is not whether but how rapidly the laser's wavelength can be scanned between its four output colors. An intracavity acousto-optic modulator will be used to attempt wide-bandwidth rapid scanning of the operating wavelength. An acousto-optic modulator can also be used to actively mode-lock the $\mathrm{cw}$ output of the green laser and produce pulse widths in the picosecond domain. Success with this experiment would be directly transferable to genesequencing work ongoing in the Life Sciences Division. Addition of saturable absorbers may further shorten the green pulsewidth, but group-velocity dispersion will limit the minimum pulse width to perhaps a few picoseconds. Techniques for compensation of the dispersion can be devised, but the most compelling application of a $\mathrm{Pr}^{3+}$ laser to ultrafast laser development is to exploit the fiber laser's superb beam quality and high output power to pump $\mathrm{Cr}^{3+}: \mathrm{LiSGAF}$ in its ground-state absorption band centered at $635 \mathrm{~nm}$. The tuning range of $\mathrm{Cr}^{3+}: \mathrm{LiSGAF}$ is less than that of Ti:sapphire, but the laser's bandwidth in the infrared is still enough to support pulses as short as $15 \mathrm{fs}$. 


\section{Results}

1. The photon avalanche mechanism initially suspected to be responsible for upconversion pumping of the upper laser level was definitively demonstrated by using a crossed confocal microscope arrangement to measure the up-conversion fluorescence from the $\mathrm{Pr}^{3+}$ ion as well as the infrared fluorescence from the $\mathrm{Yb}^{3+}$ ion.

2. We discovered an unusual "coupled cavity" effect in the red laser that yielded an unexpected asymmetry in the output power measured from either end of the fiber. This phenomenon has yet to be explained

3. A mathematical model based on measured optical parameters of $\mathrm{Yb}^{3+}$ and $\mathrm{Pr}^{3+}$ was developed in order to guide design of the laser. In particular, a finite-difference numerical solver of partial differential equations was used to perform calculations of the laser performance.

4. New cavity designs were developed that included the direct deposition of dielectric mirror coatings onto the fiber ends. We experienced a major disappointment with these coatings in that because of the low melting point of the ZBLAN fiber host material, only low temperature depositions of the coatings could be performed. Such coatings possess parasitic absorptions absent in high-temperature coatings and yielded enough heating due to the 860 -nm pump beam to destroy the coating. Some results were nevertheless still obtained with the blue laser, although low output power was measured owing to the high reflectivity of the blue output coupler. Additional experiments would likely lead to good performance of the blue laser; however, the high expense and multi-month turn around time of the coating recommended against this avenue of research.

5. A maximum output power of $700 \mathrm{~mW}$ at $635 \mathrm{~nm}$ was demonstrated with a slope efficiency of $56 \%$ with respect to absorbed pump power.

6. The emission spectrum of the red laser was measured and found to be several nm wide. This makes the laser inappropriate for frequency doubling until linewidth narrowing optics are included in the cavity design.

7. In a side project, a mathematical model of energy transfer phenomena in $\mathrm{Er}^{3+}$-doped silica glass was developed that demonstrated the effect of topological structure on upconversion quenching. This number experiment, which used Monte Carlo methods, demonstrated for the first time that the impurity topology can have an important effect on up-conversion dynamics.

8. A US Patent was issued in 1998 for the four-color up-conversion laser developed in this LDRD project. 


\section{Publications}

1. Gosnell, T. R., "Avalanche Assisted Upconversion in $\mathrm{Pr}^{3+} / \mathrm{Yb}^{3+}$-Doped ZBLAN Glass," Electron. Lett. 33, 411-2 (1997).

2. Binder, P.-M., Perondi, L. F., and Gosnell, T. R., "Model Showing Effect of Impurity Sub-Lattice Topology on Upconversion in $\mathrm{Er}^{3+}$-Doped Glasses," Electron. Lett. 34, 1348-9 (1998).

3. Gosnell, T. R., "Upconversion Lasers," Book Section to appear in "Compact BlueGreen Lasers," forthcoming from Cambridge University Press. 